\title{
Multi-scale decision making: challenges in engineering and environmental systems
}

\author{
Peter A. Beling
}

Published online: 7 September 2013

(C) Springer Science+Business Media New York 2013

Many systems analyses involve a variety of decisions defined over different temporal, physical, and organizational scales. Scale issues may introduce dependencies and influences between decisions that are difficult to anticipate or untangle with standard methods from optimization, decision theory, and risk management. These issues are made all the more important by the rapid proliferation of technology (sensors, communications, and computing) that supports the collection and processing of new types of data on unprecedented scales ("big data" decision making). Multi-scale decision problems arise in a variety of domains, including environmental management, manufacturing and production, service systems, and finance.

Multi-scale decision making is closely related to the topic of distributed decision making. There exists a large literature on distributed models for optimization and planning, with problems often classified in terms of the hierarchies that exist between decision makers (see, e.g., Deng and Papadimitriou 1999; Schneeweiss 2010). The artificial intelligence community also has considered distributed problems, with emphasis on machine learning problems in taxonomies defined in terms of paradigms for information exchange among agents (see, e.g., Doran et al. 1997; Weiss 1999; Brafman and Tennenholtz 2011). Recently, Wernz and Deshmukh $(2010,2012)$ have proposed a unified mathematical framework for multi-scale problems which defines decision hierarchies and information exchange in a game theoretic model.

In keeping with the theme of the journal, this special issue of Environment Systems \& Decisions explores multi-

P. A. Beling $(\bowtie)$

Department of Systems and Information Engineering, University

of Virginia, Charlottesville, VA 22903, USA

e-mail: pb3a@virginia.edu scale decision making from a decidedly more applied perspective. In practice, the context for decision making is where many of the true difficulties lie. Multi-scale problems often cut across organizations and stakeholder groups, and the texture of these environments may defy the kind of smooth information exchange and hierarchical characterizations so easily postulated in mathematical models. As an example of how multi-scale decision making can be driven by issues that arise from systems context, consider the growing importance of security in systems design and operation. A decision problem that might have been properly modeled as one of coordinating the actions of distributed elements within an organization must now be recast to account for the presence of an intelligent adversary. This in turn creates linkages in the decision problem among many new stakeholders, including the adversary itself.

Several of the principal challenges in multi-scale decision making are outlined below. In each case, references are made to the most relevant papers in the special issue.

\section{Engineering information exchange}

Various mathematical frameworks have been developed to model the exchange of information between decision makers in multi-scale environments. Zhao et al. (2008), to take an example, have studied coordinated decision making with agents that have a strictly limited ability to communicate. In practice, technology for the exchange of data is, and has been, a rapidly developing area. The big challenges lie elsewhere, in the design of mechanisms for exchanges between decision makers that convey information that is not easily codified or stored in a database. In this issue, Linkov et al. (2013) report that military decision makers 
often lack universally understood language for describing decision problems and formal methodologies for addressing them. Notions of risk also can be difficult to incorporate into multi-scale decision making, particularly for the wicked problem space (see, e.g., Seager et al. 2013). In this issue, Bridges et al. (2013) propose mental modeling as a tool for identifying gaps and formulating strategies for such problems. Collins et al. (2013) also study methods for assisting decision makers, but at entirely different scale. They consider how optimization models can integrate with visualization tools designed to support decision making by illiterate farmers. Xu and Lambert (2013) study multi-scale issues and planning aids for the management of access points for road networks.

\section{Cyber security and decision making in adversarial settings}

Traditionally, the field of cyber security has focused on the development of perimeter security solutions that attempt to keep adversaries from gaining access to networks and computing infrastructure. Recent years have seen a growing threat from supply chain and insider attacks, which may bypass such security perimeters. In this issue, Lambert et al. (2013) consider some of the multi-scale decision and risk management problems engendered by these new threats, with a focus on supply chains that cut across systems, organizations, and often nations. To deal with situations where attacks are embedded in systems, Jones and Horowitz (2012) have proposed that critical system functions should be protected directly using low-capability, high-security supervising electronics. In this issue, Jones et al. (2013) consider the problem of selecting a supervising architecture for systems aware cyber security, proposing a multi-scale methodology for integrating the efforts of experts in system functionality, cyber attacks, and adversary capability.

\section{Infrastructure and regulatory impacts on policy and decision making}

In many ways, economic relationships and infrastructure can bind together decision makers across organization, geography, and scale and timing of activity. In this issue, Walchuk and Barker (2013) study resource policy decisions in the context of interdependent and multi-scale industry and infrastructure sectors, adopting a classical input-output modeling approach to gain insight into the evolution and impact of resource shortages. Like economic and infrastructural relationships, regulations can create interdependencies between diverse decision-making entities. In this issue, Martinez et al. (2013) develop a game theoretic approach that can be used to help design regulations that incentive cooperation between decision makers.

\section{Human-machine collaborative decision making}

Recent advances in data analytics and computing technologies have created enormous capability for automated data analysis, prediction, and decision making. Naturally, there is considerable pressure to make use of this capability to obtain efficiencies or competitive advantages through integration of machine intelligence into every facet of decision making, including processes that were formerly the exclusive domain of human decision makers. Humanmachine collaborative making brings with it many new challenges. From the human perspective, a principal challenge is how to allocate accountability for decisions. Can a human be accountable for a decision that is (necessarily) based on input from a opaque software models and masses of data beyond imagination? From the machine perspective, as it were, an important challenge is to find ways to adapt decision support to the objectives of the human, which may change frequently. In this issue, Hayes et al. (2013) study the problem of recognizing, or classifying, the strategies employed by traders in financial markets on the basis of limited observation of their trades. This problem is an example of a task or activity recognition, which is a foundation component of adaptive decision support.

\section{Exploitation of domain knowledge}

Detailed domain knowledge, combined with advances in computing technology, may allow one to directly analyze or simulate complex, multi-scale systems with a fidelity that exceeds what could be extracted from game or team theoretic models. The trade-off is generally between generalizable insight and specific results that can be used for the scenario at hand. In this issue, Wangusi et al. (2013) explore the potential benefits of using simulations of various scales as input to decisions on the management of a large watershed. In a similar spirit, Mack et al. (2013) examine low-level analyses of groundwater levels as a means for informing the management of a large basin in a challenging geopolitical setting.

The papers in this special issue of Environment Systems \& Decisions span a broad range of problem domains, methods, and objectives. We hope this collection serves to illustrate the many challenges and issues associated with the important field of multi-scale decision making. 


\section{References}

Brafman RI, Tennenholtz M (2011) Learning to coordinate efficiently: a model-based approach. CoRR abs/1106.5258

Bridges TS, Kovacs D, Wood MD, Baker K, Butte G, Thorne S, Linkov I (2013) Climate change risk management: a mental modeling application. Environ Syst Decis 1-15. doi:10.1007/ s10669-013-9461-6

Collins A, Vegesana K, Seiler M, OShea P, Hettiarachchi P, McKenzie F (2013) Simulation and mathematical programming decision-making support for smallholder farming. Environ Syst Decis 1-13. doi:10.1007/s10669-013-9460-7

Deng X, Papadimitriou C (1999) Decision-making by hierarchies of discordant agents. Math Program 86(2):417-431. doi:10.1007/ s101070050096

Doran JE, Franklin S, Jennings NR, Norman TJ (1997) On cooperation in multi-agent systems. Knowl Eng Rev 12(3):309-314

Hayes R, Beling P, Scherer W (2013) Action-based feature representation for reverse engineering trading strategies. Environ Syst Decis 1-14. doi:10.1007/s10669-013-9458-1

Jones R, Luckett B, Beling P, Horowitz B (2013) Architectural scoring framework for the creation and evaluation of systemaware cyber security solutions. Environ Syst Decis 1-21. doi:10. 1007/s10669-013-9462-5

Jones RA, Horowitz B (2012) A system-aware cyber security architecture. Syst Eng 15(2):225-240 doi:10.1002/sys.21206

Lambert J, Keisler J, Wheeler W, Collier Z, Linkov I (2013) Multiscale approach to the security of hardware supply chains for energy systems. Environ Syst Decis 1-9. doi:10.1007/ s10669-013-9465-2

Linkov I, Wood M, Ditmer R, Cox A, Ross R (2013) Collective risk management: insights and opportunities for dod decision-makers. Environ Syst Decis 1-6. doi:10.1007/s10669-013-9452-7

Mack T, Chornack M, Taher M (2013) Groundwater-level trends and implications for sustainable water use in the Kabul basin,
Afghanistan. Environ Syst Decis 1-11. doi:10.1007/s10669-0139455-4

Martinez L, Thekdi S, Lambert J (2013) Modeling energy facility regulatory compliance with application to multi-scale liquefied natural gas facilities. Environ Syst Decis 1-9. doi:10.1007/ s10669-013-9457-2

Schneeweiss C (2010) Distributed decision making. Springer, Berlin. http://books.google.com/books?id=bH1acgAACAAJ

Seager T, Collier Z, Linkov I, Lambert J (2013) Environmental sustainability, complex systems, and the disruptive imagination. Environ Syst Decis 33(2):181-183. doi:10.1007/s10669-013-9449-2,

Walchuk Z, Barker K (2013) Analyzing interdependent impacts of resource sustainability. Environ Syst Decis 1-13. doi:10.1007/ s10669-013-9456-3

Wangusi N, Kiker G, Muoz-Carpena R, Henson W (2013) Improving watershed decisions using run-off and yield models at different simulation scales. Environ Syst Decis 1-17. doi:10.1007/s10669013-9454-5

Weiss G (ed) (1999) Multiagent systems: a modern approach to distributed artificial intelligence. MIT Press, Cambridge, MA

Wernz C, Deshmukh A (2010) Multiscale decision-making: Bridging organizational scales in systems with distributed decisionmakers. Euro J Oper Res 202(3):828 - 840. doi:10.1016/j.ejor. 2009.06.022. http://www.sciencedirect.com/science/article/pii/ S0377221709004913

Wernz C, Deshmukh A (2012) Unifying temporal and organizational scales in multiscale decision-making. Euro J Oper Res 223(3): 739-751. doi:10.1016/j.ejor.2012.06.038, http://www.science direct.com/science/article/pii/S0377221712004936

Xu J, Lambert J (2013) Distributed travel time savings of a multiscale transportation access management program. Environ Syst Decis 1-14. doi:10.1007/s10669-013-9459-0

Zhao Y, Patek SD, Beling PA (2008) Decentralized Bayesian search using approximate dynamic programming methods. Trans Syst Man Cyber Part B 38(4):970-975. doi:10.1109/TSMCB.2008.928180 